\title{
LACK OF CORRELATION OF THE SERUM 25(OH) VITAMIN D LEVELS WITH THE GLYCATED HEMOGLOBIN A1C AND THE LIPID PROFILE IN TYPE 2 DIABETES PATIENTS ON ORAL ANTIDIABETIC DRUGS - PRELIMINARY DATA
}

\author{
D. Bakalov ${ }^{1}$, M. Boyanov ${ }^{1}$, A. Tsakova ${ }^{2}$ \\ ${ }^{1}$ Clinic of Endocrinology and Metabolism, Department of Internal Medicine, \\ University Hospital Alexandrovska, Medical University - Sofia \\ ${ }^{2}$ Department of Clinical Laboratory and Clinical Immunology, \\ University Hospital Alexandrovska, Medical University - Sofia
}

Summary. Data from different studies correlating the serum 25(OH)D levels with the metabolic and glycemic parameters in type 2 diabetes patients are still varying. The objective if this study was to describe the correlation between serum 25(OH)D levels and some metabolic parameters in Bulgarian type 2 diabetes patients on oral antidiabetic drugs. One hundred type 2 diabetes patients participated -56 men and 44 women. The mean age and diabetes duration of the women was 59.0 and 9.8 years, of the men -58.0 and 7.7 years respectively. Complete patient history was taken and physical examination was performed (body weight and height, waist circumference). Body composition was measured on a leg-to-leg body impedance analyzer (TBF-215, Tanita Corp., Tokyo, Japan). Serum levels of vitamin $D$ were measured by electro-hemi-luminescent detection as 25-(OH) D Total (ECLIA, Elecsys 2010, Roche Diagnostics, Switzerland). Glycated hemoglobin A1c was measured on a NycoCard reader (Alere ${ }^{\mathrm{TM}}$ ). Total, HDL-cholesterol (direct) and triglycerides were analyzed on a Cobas Integra 400+ analyzer. Correlation analysis was performed on a SPSS 13.0 for Windows platform and included 10 curves. The data were first analyzed for the group as a whole and then separately for men and women as well as in the different vitamin $\mathrm{D}$ tertiles. The mean serum $25-\mathrm{OH}$-vitamin D levels were $23.8 \pm 12.1 \mathrm{nmol} / \mathrm{l}$ in women and $33.3 \pm 20.0 \mathrm{nmol} / \mathrm{l}$ in men. We were unable to find any statistically significant correlation between serum $25(\mathrm{OH})$ vitamin 
$\mathrm{D}$ and the serum lipids (cholesterol profile and triglycerides). On the contrary, there was a weak correlation with the glycated hemoglobin A1c (cubic model, $R^{2}=0.178$, $p=0.05$ ) and the BMI (inverse model, $R^{2}=0.101, p=0.038$ ). The sub-analyses (men versus women or according to tertiles of vitamin $\mathrm{D}$ ) did not produce any additional information. The influence of vitamin D on the parameters of the metabolic control in type 2 diabetes is very weak on an individual level. It might be only demonstrated in large epidemiological surveys. analysis

Key words: Diabetes mellitus type 2, 25(OH) vitamin D, metabolic control, correlation

\section{INTRODUCTION}

- 2 Diabetes has grown to an epidemic worldwide due to sedentary lifestyle, over-nutrition and obesity. Diabetes mellitus and vitamin D deficiency / insufficiency are often coexisting in the same subjects. This has led to epidemiological studies looking for and finding a statistical association between type 2 diabetes and vitamin $\mathrm{D}$ deficiency. This association was confirmed in both directions. Observational studies show a consistent association between low vitamin D status and prevalent type 2 diabetes [9-11, 25]. Vitamin D levels are predictive of future glycemic status and insulin resistance [1, 7, 8, 24]. There is a relationship between vitamin D and hyperglycemia in older people [21]. Some studies did not find any significant association between the vitamin $D$ status and the glycemic control, plasma lipids, blood pressure [15, 22], while others studies did find a weak one $[12,13,16]$. Moreover, some interventional studies with vitamin D supplements showed an improvement in insulin sensitivity, insulin secretion and some markers of metabolic control $[4,8,17,19]$, which was not the case in other studies [14, 23]. Many researchers support the direct role of vitamin $D$ in the pathogenesis of type 2 diabetes $[5,20,26]$.

$21.3 \%$ of the Bulgarian population have vitamin D insufficiency and $54.5 \%$ - vitamin $D$ deficiency [3]. Post hoc analyses show that low vitamin $D$ is more common in the urban population and in the presence of obesity. In a previous study we were able to prove that vitamin $\mathrm{D}$ deficiency was even more prevalent in type 2 diabetes patients on oral drugs than in the general Bulgarian population [2]. Practically all diabetics in our study had low plasma vitamin D.

\section{AIM AND OBJECTIVES}

The aim of the present study was to describe the correlation between serum 25(OH)D levels and the metabolic parameters in the same above mentioned group of Bulgarian type 2 diabetes patients on oral antidiabetic drugs. 


\section{PATIENTS AND METHODS}

This was a cross-sectional observational study conducted from November till May for two consecutive years. The time period of low sunlight exposure was selected to increase the sensitivity in detecting vitamin $D$ insufficiency. The following inclusion criteria were applied: 1 . Confirmed diagnosis of type 2 diabetes with a duration of $\geq$ one year in subjects from both sexes aged $\geq 50$ years, 2. treatment with oral antidiabetic drugs (OADs), and 3. no prior use of vitamin D containing preparations. $80 \%$ of the participants were taking metformin, and $70 \%$ were on dual oral antidiabetic therapy. The exclusion criteria included: 1. past or present insulin treatment, 2. conditions interfering with the bioavailability of vitamin $D$ - malabsorption, liver cirrhosis, renal failure, administration of anticonvulsants or corticosteroids, known hyper- or hypoparathyroidism, uncontrolled hyper- and hypothyroidism. The study protocol was approved by the Scientific Council and Ethic Committee at the Medical University Sofia. All patients signed informed consent before any study procedure was started.

Medical history was taken and a thorough physical examination was performed. All measurements were performed in the fasting state between 08.00 and 09.00 a.m. Height (in $\mathrm{cm}$ ), weight (in $\mathrm{kg}$ ) and body mass index (in $\mathrm{kg} \mathrm{x} \mathrm{m}^{2}$ ) were measured on a leg-to-leg body impedance analyzer in the standing position with light clothing (Tanita TBF-215; Tanita Inc., Japan). Waist circumference was measured as an index of visceral obesity.

Routine blood biochemistry was performed on a Cobas Integra 400+ analyzer (total cholesterol and HDL direct, triglycerides), glycated hemoglobin A1c - on a NycoCard reader (Alere $\left.{ }^{\mathrm{TM}}\right)$. Serum $25-(\mathrm{OH})$ - Vitamin D was measured by electro-hemi-luminescent detection (ECLIA method) as 25(OH)D Total (Elecsys 2010 analyzer, Roche Diagnostics, Switzerland). Vitamin D insufficiency was defined as $25(\mathrm{OH}) \mathrm{D}$ levels between 25.0 and $49.9 \mathrm{nmol} / /$, and deficiency - as levels $<25.0 \mathrm{nmol} / /$ according to the Bulgarian guideline for the diagnosis and treatment of vitamin $D$ insufficiency [18].

Statistical analysis was done on the SPSS 13.0 for Windows platform (SPSS Inc., Chicago, IL, USA). The metabolic and anthropometric parameters were tested for normal distribution. Correlation analyses included serum $25(\mathrm{OH})$-vitamin $\mathrm{D}$ as the independent variable and the metabolic and anthropometric parameters as the dependent variables. 10 regression curves were tested and the best fitting / most significant ones were selected (linear, quadratic, cubic, exponential, power, S, growth, inverse, logarithmic, compound) . The data were first analyzed for the study population as a whole and then separately for men and women as well as subdivided into tertiles of serum vitamin $D$.

\section{RESULTS}

One hundred type 2 diabetes patients participated in this study (56 men and 44 women). The mean age of the female participants was 59.0 years and of the male participants -58.0 years. The mean diabetes duration in women was $9.8 \pm 6.3$ years and $7.7 \pm 4.5$ years - in men. The most important anthropometric and laboratory parameters are shown separately for women and men in Table 1 (mean values \pm SD, 
minimum - maximum range). The mean serum 25(OH)D levels were lower than the country specific means in both genders -23.8 versus $36.3 \mathrm{nmol} / \mathrm{l}$ in women and 33.3 versus $41.5 \mathrm{nmol} / \mathrm{l}$ in men [3].

The regression formulas including lipids and waist circumference as dependent variables and $25(\mathrm{OH}) \mathrm{D}$ as the independent one did not reach statistical significance. Only the correlation between $25(\mathrm{OH}) \mathrm{D}$, BMI and HbA1c was of statistical significance (Table 2). Similar results were observed if data were analyzed separately in men and women (data not shown).

Table 1. The most important parameters of the diabetic study population are shown

\begin{tabular}{|c|c|c|c|c|c|c|}
\hline \multirow[b]{2}{*}{ Parameter } & \multicolumn{2}{|c|}{ Whole study population, $\mathrm{n}=100$} & \multicolumn{2}{|c|}{ Women $(n=44)$} & \multicolumn{2}{|l|}{ Men $(n=56)$} \\
\hline & $\begin{array}{c}\text { Range } \\
\text { (min.-max.) }\end{array}$ & Mean \pm SD & $\begin{array}{c}\text { Range } \\
\text { (min.-max.) }\end{array}$ & Mean \pm SD & $\begin{array}{c}\text { Range } \\
\text { (min.-max.) }\end{array}$ & Mean \pm SD \\
\hline $\mathrm{BMI}, \mathrm{kg} / \mathrm{m}^{2}$ & $16.8-53.6$ & $33.9 \pm 7.0$ & $17.3-53.6$ & $33.2 \pm 8.5$ & $16.8-49.6$ & $33.8 \pm 8.1$ \\
\hline $\begin{array}{l}\text { Waist circum- } \\
\text { ference, cm }\end{array}$ & $68.0-145.0$ & $111.9 \pm 16.4$ & $75.1-128.0$ & $100.7 \pm 22.6$ & $82.4-145.0$ & $\begin{array}{c}117.2 \pm \\
25.3\end{array}$ \\
\hline $\mathrm{HbA} 1 \mathrm{c}, \%$ & $5.1-12.0$ & $7.8 \pm 1.8$ & 3.8-11.8 & $7.6 \pm 2.1$ & $3.9-12.0$ & $7.7 \pm 2.1$ \\
\hline $\begin{array}{l}\text { Total choles-terol, } \\
\mathrm{mmol} / \mathrm{l}\end{array}$ & $2.47-7.58$ & $4.83 \pm 1.12$ & $1.05-7.58$ & $4.95 \pm 1.25$ & $1.16-7.49$ & $4.56 \pm 1.32$ \\
\hline $\begin{array}{l}\text { LDL-choles-terol, } \\
\mathrm{mmol} / \mathrm{l}\end{array}$ & $0.70-5.45$ & $2.72 \pm 1.01$ & $0.76-5.15$ & $2.95 \pm 0.97$ & $0.70-5.45$ & $2.47 \pm 1.00$ \\
\hline $\begin{array}{l}\text { HDL-choles-terol, } \\
\mathrm{mmol} / /\end{array}$ & $0.69-2.42$ & $1.21 \pm 0.37$ & $0.41-2.42$ & $1.29 \pm 0.45$ & $0.32-2.12$ & $1.12 \pm 0.36$ \\
\hline $\begin{array}{l}\text { Triglycerides, } \\
\mathrm{mmol} / /\end{array}$ & $0.78-6.39$ & $2.07 \pm 1.11$ & $0.78-4.06$ & $1.80 \pm 0.84$ & $0.78-6.39$ & $2.39 \pm 1.42$ \\
\hline $\begin{array}{l}\text { Serum 25-OH vit. D, } \\
\text { nmol/l }\end{array}$ & $7.5-85.1$ & $27.9 \pm 15.8$ & $7.5-51.7$ & $23.8 \pm 12.1$ & $7.5-85.1$ & $33.3 \pm 20.0$ \\
\hline
\end{tabular}

Table 2. Correlation coefficients of serum $25(\mathrm{OH})$ vitamin D levels as independent variable with the glycated hemoglobin, lipids, BMI and waist circumference as dependent variables

\begin{tabular}{|l|c|c|c|}
\hline Correlated parameter & Best fitting model & Pearson's R square & Significance, $\mathbf{p}$ \\
\hline Body mass index, $\mathrm{kg} / \mathrm{m}^{2}$ & Inverse & 0.101 & 0.038 \\
\hline Waist, cm & Quadratic & 0.034 & n.s. \\
\hline Glycated hemoglobin A1c, \% & Cubic & 0.178 & 0.050 \\
\hline Total cholesterol, mmol/l & Cubic & 0.071 & n.s. \\
\hline LDL-cholesterol, mmol/l & Cubic & 0.033 & n.s. \\
\hline HDL-cholesterol, mmol/l & Cubic & 0.066 & n.s. \\
\hline Triglycerides, mmol/l & Quadratic & 0.048 & \\
\hline
\end{tabular}


We further subdivided our participants into tertiles according to their $25(\mathrm{OH}) \mathrm{D}$ level (Fig. 1). The BMI, HbA1c and serum lipid values did not show any steady trend according to the different vitamin $\mathrm{D}$ levels - the highest values were found in the middle tertile of 25(OH)D (Fig. 1 and 2).

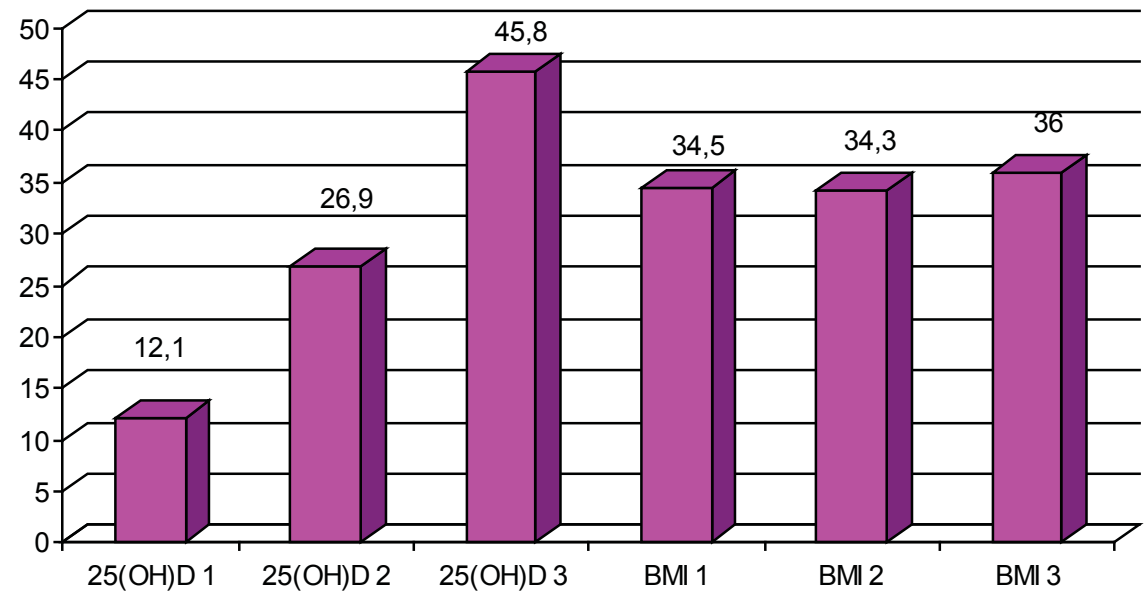

Fig. 1. Tertiles of serum 25(OH)D in the study group - mean values shown on the left three columns. The mean BMI values in $\mathrm{kg} / \mathrm{m}^{2}$ are shown in the right three columns according to the vitamin $\mathrm{D}$ status ( 1 being the lowest vitamin $D$ tertile, 3 being the highest one)

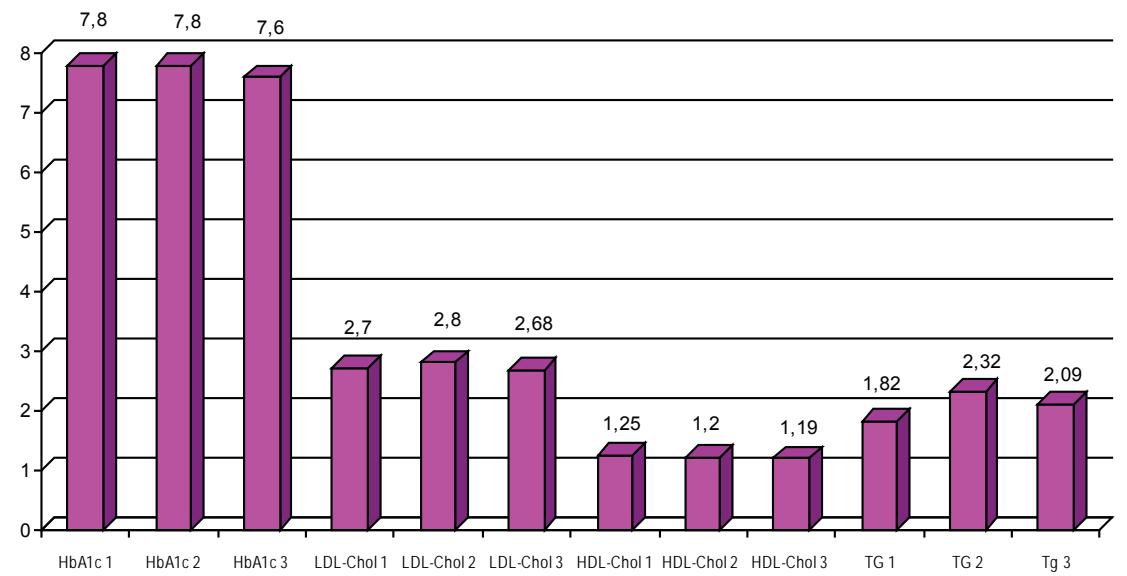

Fig. 2. The mean lipid values in the three tertiles according to the vitamin $D$ status are shown ( 1 being the lowest vitamin $D$ tertile, 3 being the highest one; TG-triglycerides) 


\section{DISCUSSION}

The hypothesis of a possible interplay between the vitamin $D$ status and the metabolic control in type 2 diabetes patients has recently found a number of supporting evidence coming from diabetics as well as non-diabetics with features of the metabolic syndrome. Data from 292 postmenopausal women aged $50-79$ years in the Women's Health Initiative showed an inverse association of higher $25(\mathrm{OH}) \mathrm{D}$ concentrations with $\mathrm{BMI}$, waist circumference, triglycerides and triglycerides/HDLcholesterol ratio while not with LDL and HDL-cholesterol, insulin or plasma glucose [6]. In a cross-sectional sample of 250 overweight and obese adults of different ethnicities modest inverse associations of vitamin D3 with body weight, $(r=-0.21)$, BMI $(r=-0.18)$, waist $(r=-0.14)$ and $\mathrm{HbA1c}(r=-0.16)$ were registered [16]. Retrospective data from 276 Korean type 2 diabetes patients showed that serum $25(\mathrm{OH}) \mathrm{D}$ was significantly related to triglycerides, LDL-cholesterol and $\mathrm{HbA1c}$ [25]. A cross-sectional study in 8018 nonsmoking and 2087 smoking subjects showed a significant increase in serum total cholesterol, HDL- and LDL-cholesterol, and a significant decrease in serum LDL/HDL-cholesterol ration and triglycerides across increasing serum $25(\mathrm{OH})$ D quartiles [12].

In our study sample we were unable to prove any significant association between serum 25(OH)D and the cholesterol profile and triglycerides. However, the correlation of $25(\mathrm{OH}) \mathrm{D}$ to $\mathrm{HbA} 1 \mathrm{c}$ and $\mathrm{BMI}$ was statistically significant, although of borderline clinical significance. These findings might be attributed to two main factors: First, our study sample was very small and therefore not powered enough to detect slight differences due to the vitamin D status; and secondly, the association of $25(\mathrm{OH}) \mathrm{D}$ with markers of the metabolic syndrome might be so weak that it is hard to prove and is therefore of no practical clinical relevance.

The contribution of the vitamin $D$ status to the markers of the metabolic control is shown to be very weak in other studies also. In the study by A-T. McGill et al. [16] the variability of $\mathrm{HbA} 1 \mathrm{c}$ attributable to $25(\mathrm{OH}) \mathrm{D}$ was $2.6 \%$, and in the study by $\mathrm{J} R$ $\mathrm{Yu}$ [25] it was $2.4 \%$. The same is observed with the serum lipids. In the study by S. Chako [6] the R2 for the association of $25(\mathrm{OH}) \mathrm{D}$ with the triglycerides was $0.01(1 \%$ variability of TG levels due to the vitamin D status) and it was 0.019 (1.9\% variability) in the study by J. R. Yu [25]. The respective contribution of the variation in $25(\mathrm{OH}) \mathrm{D}$ to the LDL-cholesterol variability was $4.7 \%$ in the latter study [25]. A stratification of blood lipids according to the vitamin D tertiles was applied in 108711 patients coming from the general population but data were consistent only if the highest tertile $(25(\mathrm{OH}) \mathrm{D} \geq 30 \mathrm{ng} / \mathrm{dl})$ was compared to the lowest one $(25(\mathrm{OH}) \mathrm{D}<20 \mathrm{ng} / \mathrm{dl})$ [22]. There are also studies which were unable to find any impact of vitamin $D$ deficiency on the clinical metabolic status and biomarkers of inflammation in type 2 diabetes [15]. 


\section{CONCLUSION}

The impact of the vitamin D status on the Hba1c and lipid profile in type 2 diabetes patients is possibly very weak. Large cross-sectional and interventional studies are needed to confirm these relationships. From the clinician's point of view serum $25(\mathrm{OH}) \mathrm{D}$ levels cannot be regarded as a relevant factor contributing to the metabolic control in type 2 diabetes patients on oral antidiabetic drugs.

\section{REFERENCES}

1. Al-Shoumer, K. A. et al. Does insulin resistance in type 2 diabetes alter vitamin D status? Prim. Care Diabetes, 7, 2013, № 4, 283-7.

2. B a kalov, D., M. Boyanov, A. Tsakova, et al. Prevalence of deficiency and insufficiency of vitamin D in patients with type 2 diabetes mellitus on oral antidiabetic drugs. - Endocrinologia, 18, 2013, № 1, 19-26. (article in Bulgarian).

3. Borissova, A. M. et al. Frequency of vitamin D deficiency and insufficiency in the Bulgarian population $\geq 20-80$ years. - Endocrinologia, 17, 2012, № 3, 122-134 (article in Bulgarian).

4. B o r i s s o v a, A-M., Ts. Tankova, G. Kirilov, et al. The effect of vitamin D3 on insulin secretion and peripheral insulin sensitivity in type 2 diabetic patients. - Int. J. Clin. Pract., 57, 2003, № 4, 258-261.

5. Calle, C., M. Begoña et M. García-Arencibia. Genomic actions of 1,25-dihydroxyvitamin D3 on insulin receptor gene expression, insulin receptor number and insulin activity in the kidney, liver and adipose tissue of streptozotocin-induced diabetic rats. - BMC Molecular Biology, 9, 2008, 65.

6. Ch a ck o, S. A., Y. Song, J. E. Manson, et al. Serum 25-hydroxyvitamin D concentrations in relation to cardiometabolic risk factors and metabolic syndrome in postmenopausal women. - Am. J. Clin. Nutr., 94, 2011, № 1, 209-217.

7. Ganji, V. et al. Serum 25-hydroxyvitamin D concentrations are associated with prevalence of metabolic syndrome and various cardiometabolic risk factors in US children and adolescents based on assay-adjusted serum 25-hydroxyvitamin D data from NHANES 2001-2006. - Am. J. Clin. Nutr., 94, 2011, №1, 225-233.

8. G e orge, P. S., E. R. Pearson et M. D. Witham. Effect of vitamin D supplementation on glycaemic control and insulin resistance: a systematic review and meta-analysis. - Diabet. Med., 29, 2012, № 8, e142-50.

9. Gradinaru, D. et al. Vitamin D Status and Oxidative Stress Markers in Elderly with Impaired Fasting Glucose and Type 2 Diabetes Mellitus. - Aging Clin. Exp. Res., 24, 2012, № 6, 595-602.

10. Hu s e mo e n, L. L. et al. Serum $25(\mathrm{OH}) \mathrm{D}$ and type 2 diabetes association in a general population: a prospective study. - Diabetes Care, 35, 2012, № 8, 1695-1700.

11. Hutchinson, M. S. et al. Serum 25-hydroxyvitamin D levels in subjects with reduced glucose tolerance and type 2 diabetes - the Tromsø OGTT-study. - Int. J. Vitam. Nutr. Res., 81, 2011, № 5, 317-327.

12. Jorde, R. et al. High serum 25-hydroxyvitamin D concentrations are associated with a favorable serum lipid profile. - Eur. J. Clin. Nutr., 64, 2010, № 12, 1457-1464.

13. Jorde, R. et G. Grimnes. Vitamin D and metabolic health with special reference to the effect of vitamin D on serum lipids. - Prog. Lipid Res., 50, 2011, № 4, 303-312.

14. Kampmann, U. et al. Effects of 12 weeks high dose vitamin D3 treatment on insulin sensitivity, beta cell function, and metabolic markers in patients with type 2 diabetes and vitamin $D$ insufficiency - a double-blind, randomized, placebo-controlled trial. - Metabolism, 63, 2014, № 9, 1115-1124. 
15. Lu o, C. et al. Hypovitaminosis D in Chinese type 2 diabetes: Lack of impact on clinical metabolic status and biomarkers of cellular inflammation. - Diab. Vasc. Dis. Res., 6, 2009, № 3, 194-199.

16. McGill, A-T. et al. Relationships of low serum vitamin D3 with anthropometry and markers of the metabolic syndrome and diabetes in overweight and obesity. - Nutr. J., 7, 2008, 4.

17. Menon, R. K. et al. The effects of vitamin D2 or D3 supplementation on glycaemic control and related metabolic parameters in people at risk of type 2 diabetes: protocol of a randomised doubleblind placebo-controlled trial. - BMC Public Health, 13, 2013, 999.

18. Min is try of Health. Guideline for the diagnosis, prevention and treatment of vitamin D deficiency and insufficiency. Bulgarian Societies of Rheumatology, Endocrinology, Pediatrics and Clinical Laboratory, 2013 (in Bulgarian).

19. N a s ri , H. et al. Impact of oral vitamin D (cholecalciferol) replacement therapy on blood pressure in type 2 diabetes patients; a randomized, double-blind, placebo controlled clinical trial. - J. Nephropathol., 3, 2014, № 1, 29-33.

20. Pa l o $\mathrm{mer}$, X. et al. Role of vitamin $\mathrm{D}$ in the pathogenesis of type 2 diabetes mellitus. - Diab. Obes. Metab., 10, 2008, № 3, 185-197.

21. Pittas, A.G., J. Lau, F.B. Hu et B. Dawson-Hughes. The role of vitamin D and calcium in Type 2 Diabetes. A systematic review and meta-analysis. - J. Clin. Endocrinol. Metab., 92, 2007, № 6, 2017-2029.

22. Ponda, M. P. et al. Vitamin D may not improve lipid levels: A serial clinical laboratory data study. - Circulation, 126, 2012, № 3, 270-277.

23. Toxqui, L. et al. Changes in blood pressure and lipid levels in young women consuming a vitamin D-fortified skimmed milk: A randomised controlled trial. - Nutrients, 5, 2013, № 12, 4966-4977.

24. Yilmaz, H. et al. Is vitamin D status a predictor of glycaemic regulation and cardiac complication in type 2 diabetes mellitus patients? - Diab. Metab. Syndr., 6, 2012, № 1, 28-31.

25. $\mathrm{Yu}, \mathrm{J}$. R. et al. Serum vitamin $\mathrm{D}$ status and its relationship to metabolic parameters in patients with type 2 diabetes mellitus. - Chonnam Med. J., 48, 2012, № 2, 108-115.

26. $\mathrm{Zh} \mathrm{a} \mathrm{o,} \mathrm{Y.} \mathrm{et} \mathrm{al.} \mathrm{Association} \mathrm{of} \mathrm{vitamin} \mathrm{D}$ receptor gene polymorphisms with metabolic syndrome: a case-control design of population-based cross-sectional study in North China. - Lipids Health Disease, 13, 2014, 129.

Sponsorship: This study was sponsored by the Scientific Council of the Medical University of Sofia, Bulgaria; Grant № 60 / 2011 , Project № 46.

\author{
Corresponding author: \\ Prof. Mihail Boyanov, PhD \\ Clinic of Endocrinology \\ University Hospital Alexandrovska \\ 1, G. Sofiyski str. \\ BG - 1431 Sofia \\ Fax: +35929230 779 \\ e-mail: mihailboyanov@yahoo.com
}

